\title{
OPEN Anterior cruciate ligament remnant cells have different potentials for cell differentiation based on their location
}

\begin{abstract}
Jin Kyu Lee ${ }^{1,6}$, Sungsin Jo ${ }^{2,6}$, Young Lim Lee ${ }^{2}$, Hyosun Park ${ }^{2,3}$, Jun-Seob Song ${ }^{4}$, Il-Hoon Sung ${ }^{1}$ \& Tae-Hwan $\operatorname{Kim} \mathbb{1}^{2,5^{*}}$

Histological and cytological observations of the human anterior cruciate ligament (ACL) had been described, but the differentiation potency based on their location is still unknown. To determine and compare proliferation and differentiation potential of cells derived from distal and middle thirds of the $A C L$ remnant, $A C L$ remnant was initially marked at the distal third (within $10 \mathrm{~mm}$ from the tibial insertion) and middle third (between $10-20 \mathrm{~mm}$ from the tibial insertion) and then dissected. Both the middle and distal third regions of $\mathrm{ACL}$ remnant were analyzed using $\mathrm{CD}_{3} 4^{+}$cell counting. Cell proliferation rate did not differ in both middle and distal third regions of ACL remnant, but they showed different characteristics in cell differentiation depending on their location. The distal third region of the $\mathrm{ACL}$ remnant had a tendency for chondrogenic differentiation with higher expression of $\mathrm{CD} 34^{+}$cells. On the other hand, the middle third region of ACL remnant had a strong tendency for osteogenic and ligamentous differentiation. Characteristics of the ACL remnant tissues should be considered when performing remnant-preserving or harvesting $\mathrm{ACL}$ remnants for tissue engineering.
\end{abstract}

Anterior cruciate ligament reconstruction (ACLR) is one of the most common surgical procedures in the field of orthopaedic sports medicine, with more than 130,000 procedures performed annually in the United States alone $^{1,2}$. It has been well documented that a completely ruptured ACL does not spontaneously heal because of poor vascular supply and an unfavourable intra-articular environment ${ }^{3}$. Given the importance of its biomechanical function, surgical treatment is generally accepted as the standard procedure for restoring knee stability. In most cases, non-augmented primary repair has been unsuccessful, and therefore ACL reconstruction is required ${ }^{1,4,5}$.

For surgical success, ACLR requires tendon graft healing in a surgically created bone tunnel and maturation (i.e., ligamentization) of the graft substance ${ }^{4,6-9}$. Indeed, the lack of vascularity within the tendon graft induces degeneration or micro ruptures during the early postoperative period ${ }^{10}$. To overcome these issues, tissue engineering using stem cells has been widely explored as a means to achieve early graft healing, tendon regeneration, and bone integration. Recently, reports have shown that ruptured human ACL tissues can possess numerous vascular-derived stem cells and that ACL-derived $\mathrm{CD} 34^{+}$cells can promote healing and have high expansion and multilineage differentiation potential ${ }^{7,11-13}$. Mifune et al. ${ }^{14}$ demonstrated that ACL-derived CD $34^{+}$cells contributed to tendon-bone healing after ACLR via angiogenesis and osteogenesis enhancements ${ }^{15}$. Furthermore, Matsumoto et al. ${ }^{16}$ found that incorporation of ruptured ACL tissues in autologous grafts reduced tunnel enlargement in ACLR ${ }^{16,17}$. However, with regard to their clinical application, potential advantages of remnant-derived stem cells are still questionable. Histological observations of the uninjured human ACL have shown a different composition of cells in different ACL regions: chondrocyte-like cells were predominantly found in the more distal area (approximately $10 \mathrm{~mm}$ proximal to the tibial ligament insertion), while fibroblasts were predominantly

${ }^{1}$ Department of Orthopaedic Surgery, Hanyang University Hospital, Seoul, Republic of Korea. ${ }^{2}$ Hanyang University Institute for Rheumatology Research, Seoul, Republic of Korea. ${ }^{3}$ Department of Bioenvironmental Technology, College of Natural Sciences, Seoul Women's University, Seoul, Republic of Korea. ${ }^{4}$ Department of Orthopaedic Surgery, Gangnam JS Hospital, Seoul, Republic of Korea. ${ }^{5}$ Department of Rheumatology, Hanyang University Hospital for Rheumatic Diseases, Seoul, Republic of Korea. ${ }^{6}$ These authors contributed equally: Jin Kyu Lee and Sungsin Jo. *email: thkim@hanyang.ac.kr 
found in the more proximal region (approximately $25 \mathrm{~mm}$ proximal of the tibial ligament insertion) ${ }^{18}$. Therefore, with remnant cells having the potential for expansion and multilineage differentiation, ruptured human ACL remnant tissue where the ACL contains numerous vascular-derived stem cells may show different characteristics in cell differentiation by their location. Based on these latter findings, we performed experiments to test the hypothesis that human ACL remnant cells acquired from the site of ACL rupture have different potentials in cell differentiation based on their location.

\section{Results}

Differential CD34 level in ACL remnant cells. FACS analysis and qRT-PCR data showed higher population of CD34 positive cells in the distal third region of the ACL remnants than in the middle third region, although it did not reach statistical significance (Fig. 1a,b,c). Histological analysis and immunostaining also displayed that distal third region have many blood vessels and expressing CD34 (Supp. Data 1). Moreover, there were no significant differences of surface markers between middle and distal cells (Fig. 1d) and co-expressions of CD34 with CD90 or CD105 in both (Fig. 1e), but both subpopulations were characterized by the high expression of CD90, moderate expression of CD105, and low expression of CD146. These data indicate that cell distribution in the distal of ACL remnants is more positive for CD34 expression than in the middle.

Expansion potential and basal characteristics of ACL remnant cells. Cell proliferation potential was determined using water-soluble tetrazolium salt (WST), and cell proliferation rates were comparable between the distal and middle third regions during seven days of growth. Cell proliferation rates of both cells did not differ (Fig. 2a). When basal mRNA expression levels were compared, upregulation of ALP and RUNX2 was observed in the middle third region ( $\mathrm{p}=0.004$ and $\mathrm{p}=0.629$, respectively), while upregulation of SOX9 and collagen type 2 (COL2) was observed in the distal third region ( $\mathrm{p}=0.024$ and $\mathrm{p}=0.157$, respectively) (Fig. 2b). Immunofluorescence and immunoblotting analysis further confirmed upregulation of ALP and SOX9 in the middle third and distal third regions, respectively (Fig. 2c,d). The immunoblotting results of RUNX2, SOX9, and ALP proteins were quantified and shown as Fig. 2e.

Multilineage differentiation of ACL remnant cells. Osteogenic differentiation. Remnant cells from the middle third region showed strong staining in ALP and alizarin red (ARS) (Fig. 3). In the ALP activity and ARS quantification assays, the middle third region showed significantly higher ALP expression in the first three days (Fig. 3b, upper) and higher ARS staining after 14 days (up to 21 days) of differentiation ( $<0.05$ ) (Fig. 3b, lower). When comparing osteogenic differentiation, the expression of ALP and RUNX2 proteins was more increased up to 3 days in middle third region and thereafter gradually decreased. In addition, mRNA expressions of ALP, RUNX2, and OCN at 3 days were significantly increased in the middle third region after the osteogenic differentiation stimulus was given ( $\mathrm{p}=0.015)$ (Fig. 3d). Notably, the osteogenic differentiation potential was higher in the middle third region of the ACL than in the distal third region.

Chondrogenic differentiation. Remnant cells of the distal third region showed better staining by toluidine blue and safranin $\mathrm{O}$ than the middle third region (Fig. 4a and Supp. Data 2). Although significant increases of chondrogenic markers (SOX9, ACAN, and COL2) were noted in both regions after the chondrogenic stimulus was given, the increase was greater in the distal third region compared to the middle third region $(\mathrm{p}=0.001)$ (Fig. 4b). Collectively, the chondrogenic differentiation potential was relatively higher in the distal third region than in the middle third region.

Ligamentous differentiation. Remnant cells of the middle third region showed significantly higher expression of basal ligamentous differentiation-related genes (e.g., collagen type 1 and tenasin $\mathrm{C}$ ) than those from the distal third region $(p=0.013$ and $p=0.044$, respectively) (Fig. 5a). Since Platelet-derived growth factor (PDGF) is known to facilitate ACL graft remodelling and ligamentization, PDGF was given in a dose-dependent manner to assess proliferation and collagen analysis of both middle and distal third regions. PDGF has a growth-increasing effect on the both regions (Fig. 5b). The effect of PDGF on total collagen synthesis of both the middle and distal third regions was reproducible (Fig. 5c,d); however, increased expressions of collagen type 1 (COL1) collagen type 3 (COL3) and tenasin C (TNC) were significantly greater in the middle third region compared to the distal third region in response to PDGF (Fig. 5e). In particular, elevated COL1 expression of distal third region by PDGF was confirmed by immunostaining (Fig. 5f). Therefore, the ligamentous differentiation status with PDGF stimulation was relatively higher in the middle third region than in the distal third region.

\section{Discussion}

This study demonstrated that ACL remnants have different potentials in cell differentiation based on their location. The distal third region of the ACL remnant had a strong tendency for chondrogenic differentiation compared to the middle third region, where a strong tendency for osteogenic and ligamentous differentiation was found.

Peterson et al. ${ }^{19}$ investigated the human ACL microstructure and found that the distal region of the ACL, approximately 5-10 $\mathrm{mm}$ proximal to the tibial insertion, was predominantly populated with round- to ovoid-shaped cells that resemble chondrocytes, whereas the middle third region was predominantly populated with elongated fibroblasts. In the present study, cells in the ACL distal third were more strongly stained and expressed higher mRNA protein levels for CD34 and chondrogenic differentiation markers (ACAN, collagen 2, and SOX9). This indicates the presence of more blood vessels and a stronger potential for chondrogenic differentiation as compared to cells in the middle third. On the other hand, the more proximal portion of the remnants stained and expressed higher mRNA protein levels for osteogenic (e.g., ALP) and ligamentous differentiation 
a
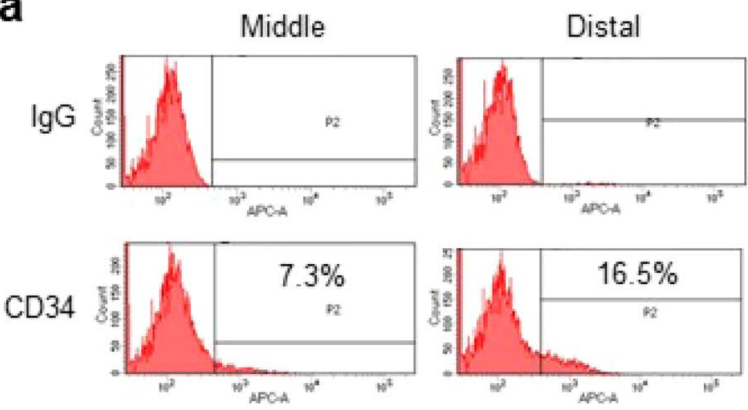

d

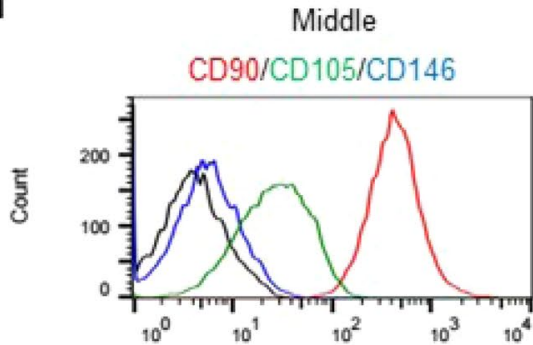

b

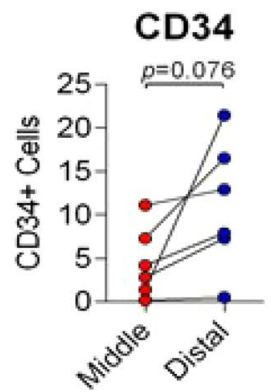

C

\section{CD34}

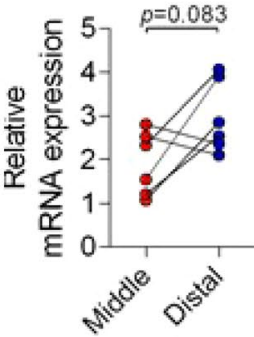

e

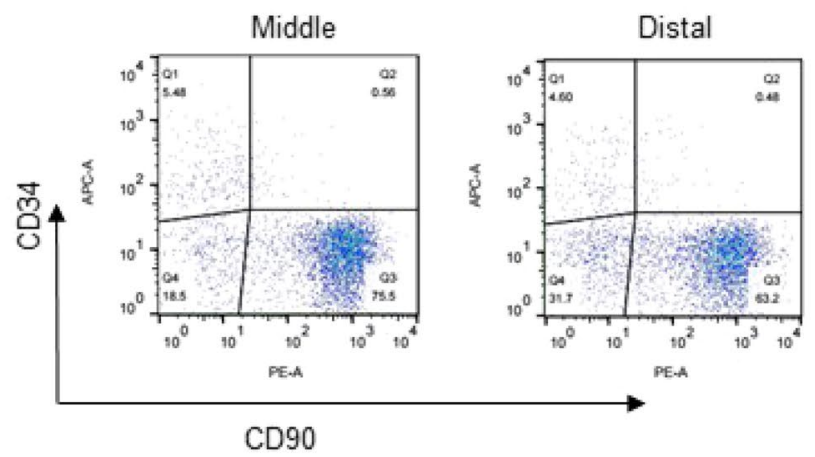

Distal
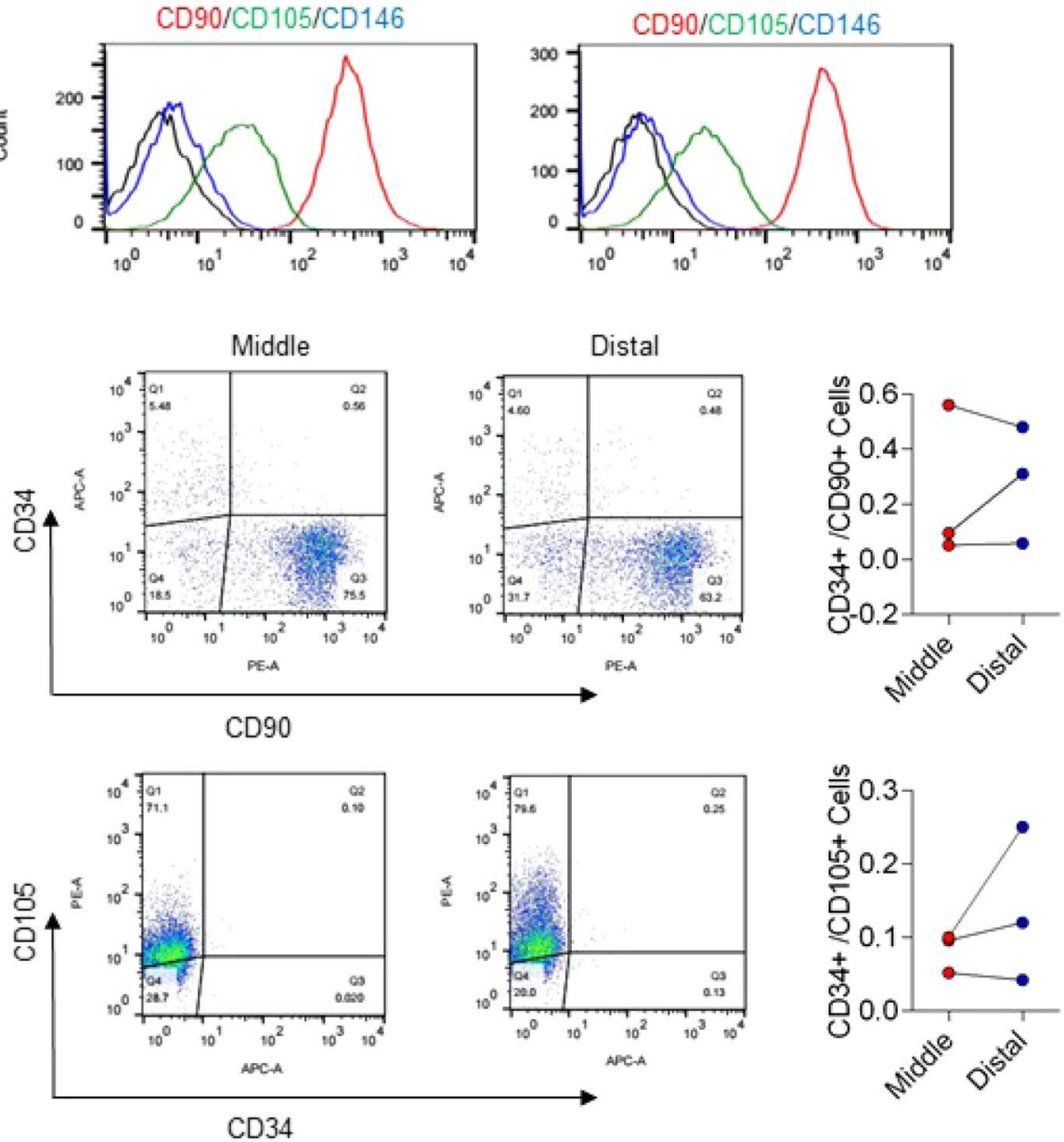

Figure 1. Differential CD34 level in cells derived from middle and distal third of ACL remnant regions. (a) CD34 levels in middle and distal cells of ACL remnant regions were analysed by FACS analysis. (b) Quantification of (a). Red, Middle ( $\mathrm{n}=6$ ); Blue, Distal $(\mathrm{n}=6)$. (c) CD34 mRNA expression was confirmed by qRT-PCR. Red, Middle $(n=6)$; Blue, Distal $(n=6)$. Representative FACS data are shown. Error bars show standard error of the mean $(\mathrm{n}=6) .{ }^{*} p<0.05$. (d) CD90, CD105, and CD146 in middle and distal cells of ACL remnant regions was analysed by FACS. Red, CD90; Green, CD105; Blue, CD146. (e) Co-expressions of CD34 with CD90 or CD105 were analysed by FACS. Red, Middle ( $\mathrm{n}=3)$; Blue, Distal $(\mathrm{n}=3)$.

markers (e.g., collagen 1, collagen 3, and TNC). Mifune et al. ${ }^{14}$ reported that ruptured ACL remnant tissue included more abundant $\mathrm{CD} 34^{+}$vascular-derived stem cells than the non-injured ACL mid substance, which further highlighted the finding that $\mathrm{CD} 34^{+}$cells had a higher potential for proliferation and multilineage differentiation. Furthermore, Matsumoto et al. ${ }^{17}$ tested for the maturation of bone-tendon integration in a dog model of ACL reconstruction and found endochondral ossification-like integration with enhanced angiogenesis in the grafts of tissue treated with CD $34^{+}$cells. In the present study, we further divided the location of ruptured ACL remnant tissue and found that $\mathrm{CD} 34^{+}$vascular-derived stem cells were more abundant in the distal third portion 
a

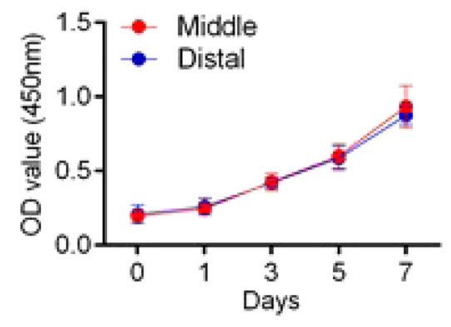

b
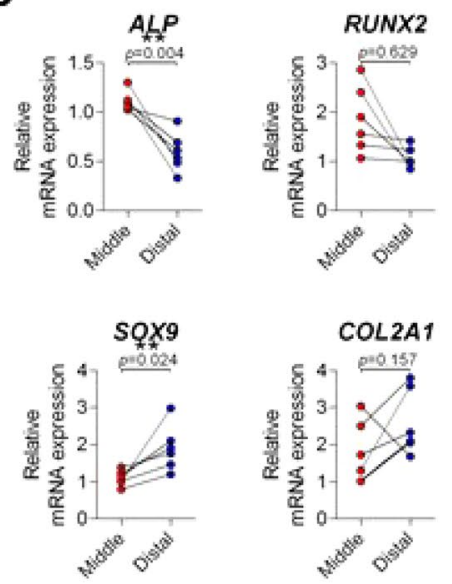

C

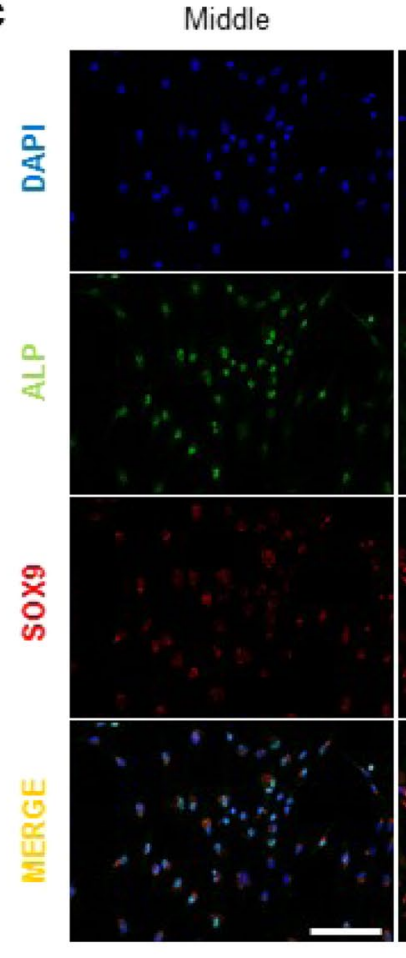

Middle

Distal

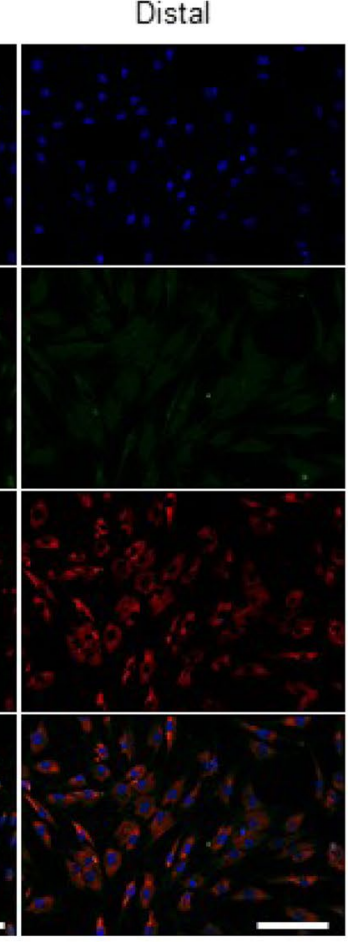

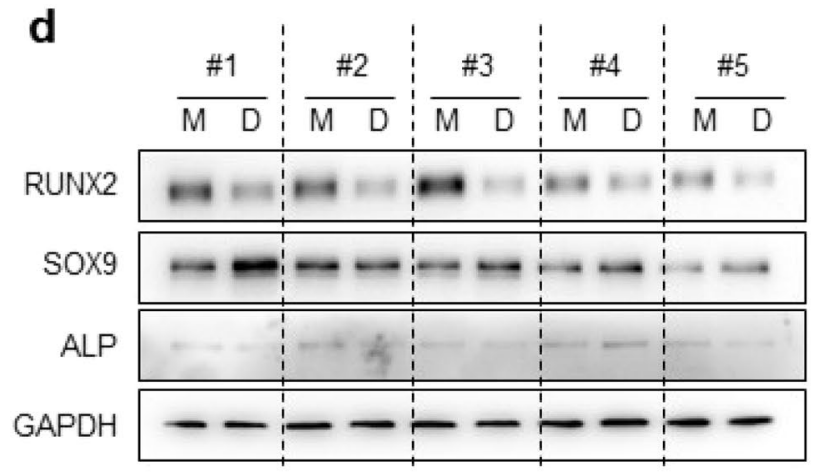

$\mathbf{e}$

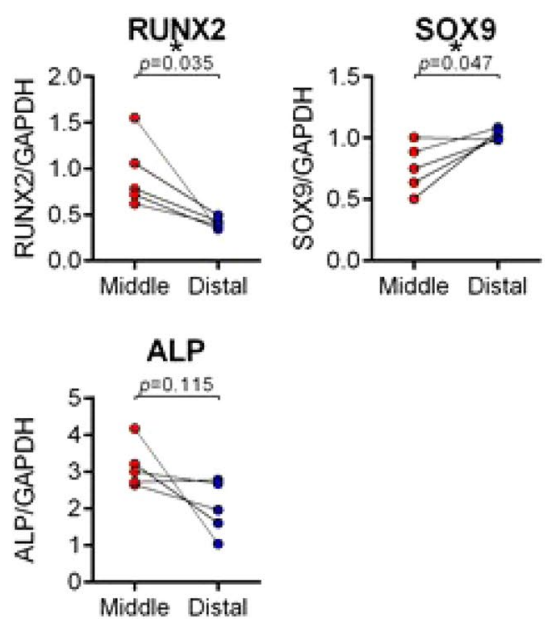

Figure 2. Comparison of basal levels in cells derived from middle and distal third of ACLR regions. (a) Cell proliferation rates were determined using a WST assay. $\mathrm{X}$ axis: days; $\mathrm{Y}$ axis: $\mathrm{OD}$ value $(450 \mathrm{~nm})$. Red, Middle $(\mathrm{n}=5)$; Blue, Distal $(\mathrm{n}=5)$. (b) mRNA expressions of ALP, RUNX2, SOX9, and COL2 were compared by qRTPCR. Red, Middle $(n=6)$; Blue, Distal $(n=6)$. (c) Expressions of ALP and SOX9 in both middle and distal cells were compared by immunofluorescence. Green, ALP-alexa-488; Red, SOX9-cy3; Blue, DAPI; Scale bar: $200 \mu \mathrm{m}$. Representative image data are shown $(\mathrm{n}=3)$. (d) Expression of RUNX2, SOX9, ALP, and GAPDH were detected by immunoblotting $(\mathrm{n}=5)$. (e) Quantification of $(\mathbf{d})$. Red, Middle $(\mathrm{n}=5)$; Blue, Distal $(\mathrm{n}=5)$. Error bars show standard error of the mean. $* p<0.05$. $* *<0.01$.

of the ACL remnant than in the middle third portion of the injured ACL substance. However, characteristics of the stem cell differentiation potential seemed to be more dependent on the location (e.g., cell type) of the remnants, rather than on the abundance of $\mathrm{CD} 34^{+}$vascular-derived cells, as the middle third portion of the remnants showed a stronger tendency for osteogenic and ligamentous differentiation even with a smaller amount of CD34 ${ }^{+}$ vascular-derived stem cells.

Remnant-preserving ACLR has gained attention due to proprioceptive function and accelerated remnant cell healing potential ${ }^{10,20-24}$. Mechanoreceptors distributed in the ACL and remnant tissue 
a
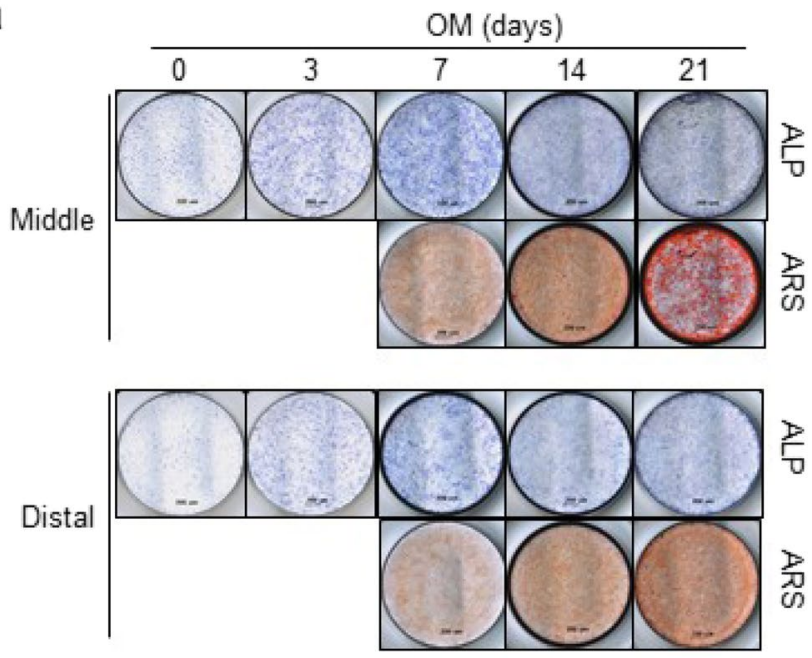

b
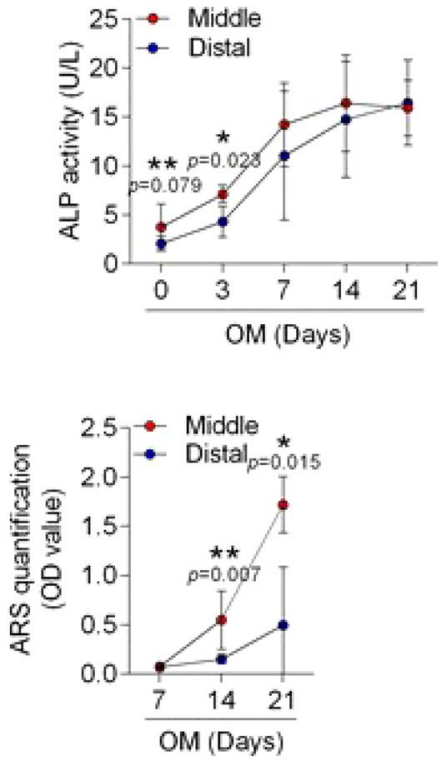

C

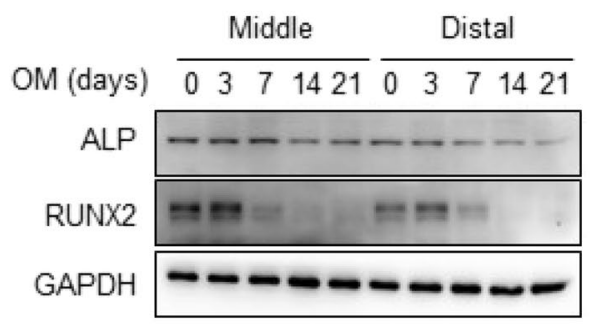

d

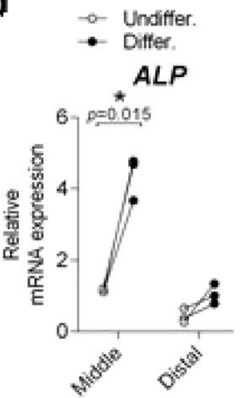

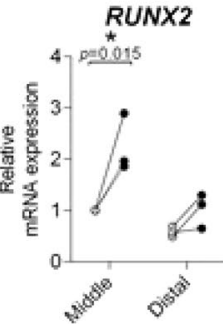

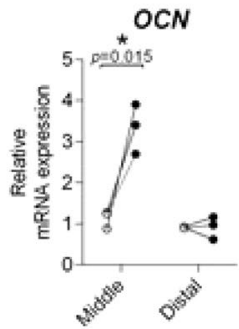

Figure 3. Comparison of osteogenic potential in cells derived from middle and distal third of ACL remnant regions. (a) Both ACL remnant cells were induced into osteogenic differentiation as determined by ALP and ARS staining on the indicated days. Representative images are shown. (b) Differentiated cells were assessed by intercellular ALP activity (upper) and quantitative ARS staining (lower). Red, Middle ( $\mathrm{n}=6$ ); Blue, Distal $(n=6)$. (c) Protein expressions in differentiated cells were detected by immunoblotting. Representative images are shown $(\mathrm{n}=3)$. (d) mRNA expression of differentiated cells at three days was determined by qRT-PCR. Osteogenic-related genes: ALP, RUNX2, and OCN. Open circle, Undifferentiation $(n=3)$; Closed circle, Differentiation $(\mathrm{n}=3)$. Error bars show standard error of the mean. ${ }^{*} p<0.05 .{ }^{* *} p<0.01$.

contribute to proprioception of the knee. Although research on mechanoreceptor regeneration and its function in an ACL-reconstructed knee is lacking, surgeons tend to preserve some of the tibial ACL remnants in order to improve knee function. Zhang et al. ${ }^{25}$ performed a randomized controlled trial for remnant-preserving ACLR in order to investigate the effect of remnant preservation on tibial tunnel enlargement using a hamstring autograft. The authors found that tibial remnant preservation can resist tibial tunnel enlargement, demonstrating that the remnant tibial side has tendon-bone healing potential. Ahn et al. ${ }^{20}$ reported on an ACLR technique using remnant preservation covering the entire graft, and they observed good clinical results with intact ACL grafts in 45 out of 48 reconstructed knees. Similarly, in a retrospective study of 218 patients from Takazawa et al ${ }^{26}$, the authors reported significantly reduced graft rupture rates in the remnant-preservation group, finding only one graft rupture out of $85 \mathrm{knees,} \mathrm{demonstrating} \mathrm{that} \mathrm{remnants} \mathrm{covering} \mathrm{the} \mathrm{entire} \mathrm{graft} \mathrm{lower} \mathrm{the} \mathrm{graft} \mathrm{rupture} \mathrm{rate.}$ There are several studies reporting second-look findings after remnant-preserving technique. Kim et al. ${ }^{27}$ assessed 66 consecutive patients who underwent second-look arthroscopy after ACLR using a hamstring autograft and found that cases with $50 \%$ or more preserved remnant showed thicker graft tissue with better synovial converage. Guo et al. ${ }^{28}$ performed second-look arthroscopy after ACLR using a bone-patellar tendon-bone allograft, and found that patients with no remnant or lower position remanant had poorer synovium coverage. Furthermore, Noh et al..$^{29}$ studied the effect of remnant-preservation on achilles allograft, and also found better synovial coverage of the allograft after remnant-preserving and retensioning ACLR. Despite numerous studies, the remnant volume or the extent of remnant preservation necessary for promoting tendon-bone healing or graft maturation is still unclear. In the present study, ACL remnant cells, regardless of their location, showed similarly high expansion and multilineage differentiation potential. However, the distal third region of the ACL remnant showed a stronger 
a

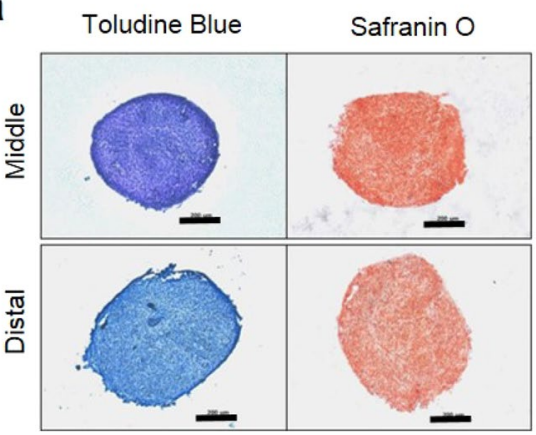

b $\rightarrow-$ Undiffer
$\rightarrow$ Differ. $\operatorname{sox} 9$
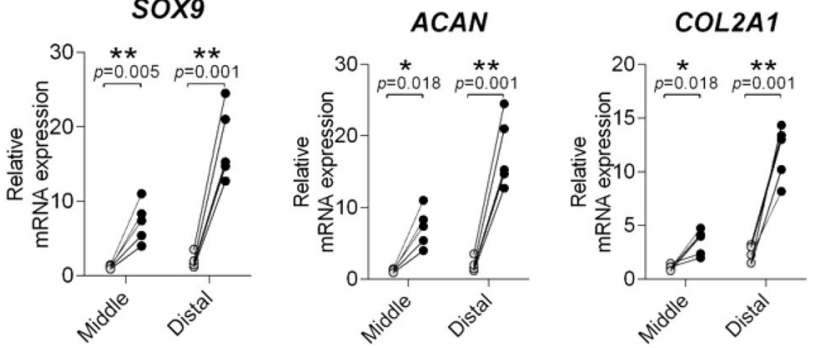

Figure 4. Comparison of chondrogenic potential in cells derived from middle and distal third of ACL remnant regions. (a) Both ACL remnant cells were induced into chondrogenic differentiation as determined by toluidine blue and safranin $O$ staining at 35 days. Representative images are shown $(n=5)$. Scale bar: $200 \mu m$. (b) mRNA expression of differentiated cells at 35 days was confirmed by qRT-PCR $(n=5)$. Chondrogenic-related genes: ACAN, COL2, and SOX9. Open circle, Undifferentiation $(n=5)$; Closed circle, Differentiation $(n=5)$. Error bars show standard error of the mean. $* p<0.05$. ** $p<0.01$.

tendency for chondrogenic differentiation compared to the middle third region, where a stronger tendency for osteogenic and ligamentous differentiation was found. During remnant-preserving ACLR or when harvesting ACL remnants for tissue engineering purposes, surgeons may consider the characteristics of ACL remnants by their location to predict the healing potential ${ }^{30}$.

The healing potential of the remnant is influenced by a number of different factors, which suggests that the characteristics of individuals should be considered ${ }^{18,25,31-34}$. Nakano et al..$^{31}$ found that ACL-derived cells from a younger group enhanced bone-tendon healing in an immunodeficient ACL reconstruction rat model. It has also been reported that ACL remnants in younger patients exhibited higher proliferation and multilineage differentiation potential. This potential decreased with age, as CD34 ${ }^{+}$cells were more prevalent in ACL remnants from younger patients. Naraoka et al..$^{32}$ demonstrated a time-dependent alteration in gene expression patterns that decrease over time in ruptured ACL tissue. Moreover, Zhang et al. ${ }^{25}$ reported that ruptured ACL remnants extracted during the early phase (within three months) of ACL injury displayed a higher proliferation and multilineage differentiation potential than remnants extracted during the chronic phase. The authors observed that $\mathrm{CD}_{3} 4^{+}$cells were more prevalent in ACL remnants from an early phase of injury compared with those from the chronic phase. Furthermore, Kirizuki et al. ${ }^{33}$ studied ACL healing potential by morphologic pattern (attachment of the remnants to surrounding tissues) and found a significantly higher number of CD $34^{+}$cells in the non-reattachment group as compared to the reattachment group.

This study has several limitations. First, ACL length differences among individuals were not considered. We uniformly applied a $10-\mathrm{mm}$ to 20 - $\mathrm{mm}$ mark from the tibial insertion as the distal and middle third boundaries. However, the average total intra-articular ligament length is approximately $32 \mathrm{~mm}$, so it should be reasonable to apply these parameters ${ }^{19,35}$. Second, excised ACL remnants may have been mixed with synovial tissues, which may have influenced the results. However, as we have devoted the best effort to removing synovial tissue macroscopically, this issue should have been minimized. Third, the anterior-posterior axis (bundles) of the ligament was not considered, and this issue may have caused heterogeneity in the tissue samples. Fourth, only ACL ruptures that occurred at the femoral attachment were included for the purpose of the study, and therefore the findings of the study cannot be generalized to all ACL injuries. In addition, although not completely transected, injury may have also occurred on the harvested remnant tissue, which may have influenced the results. However, as remnant-preserving ACLR and tissue engineering using ACL-derived stem cells are based on such injured remnants, results obtained in this study should be noteworthy. Finally, only acute ACL ruptures (within four weeks of injury) were assessed in this study. Therefore, characterization of the remnant cells in chronic or subacute situations remains unknown.

In conclusion, our results demonstrated that ACL remnants have different potentials for cell differentiation based on their location. The distal third region of the ACL remnant showed a stronger tendency for chondrogenic 
a
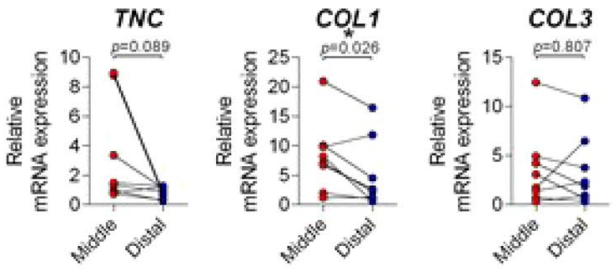

b

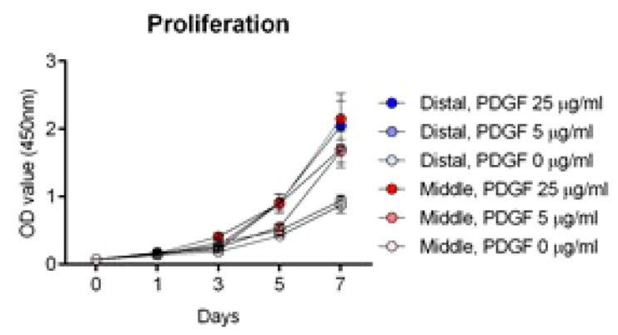

d \begin{tabular}{cccccccc} 
Days & 0 & \multicolumn{3}{c}{3} & & \multicolumn{3}{c}{7} \\
\cline { 3 - 5 }$(\mu \mathrm{g} / \mathrm{ml})$ & & 0 & 5 & 25 & 0 & 5 & 25
\end{tabular}
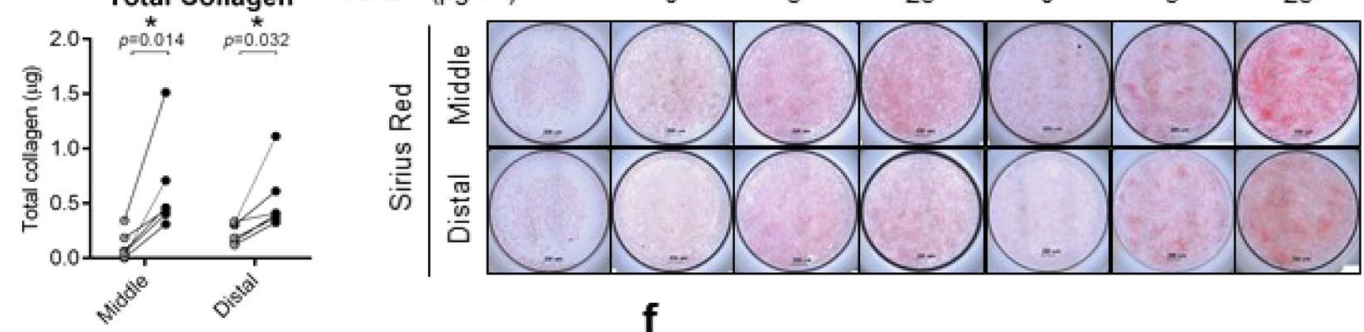

f
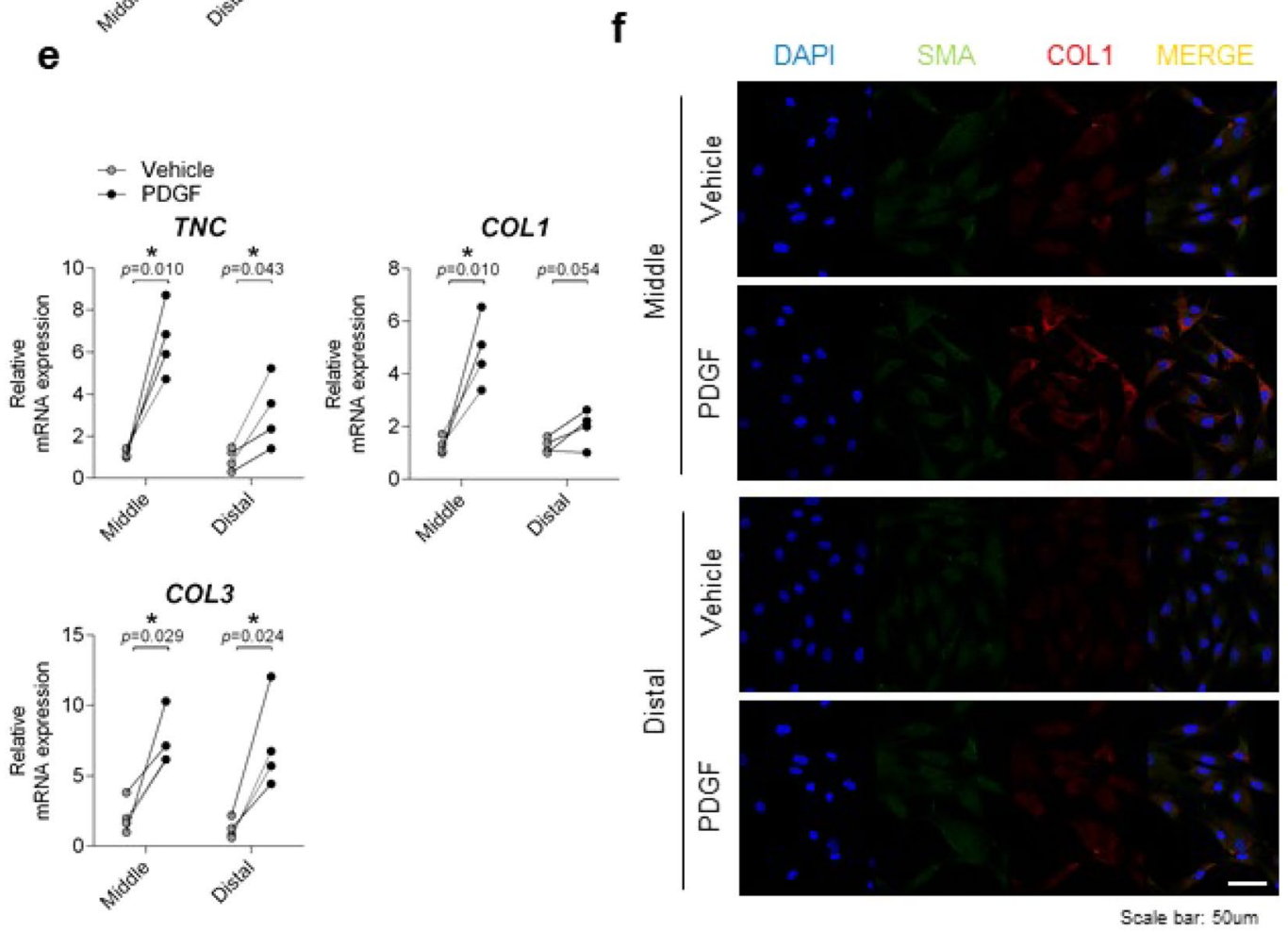

Figure 5. Comparison of ligamentous potential in cells derived from middle and distal third of ACL remnant regions. (a) mRNA expressions were compared by qRT-PCR. Ligamentous-related genes: COL1, COL3, and TNC. Red, Middle $(\mathrm{n}=8)$; Blue, Distal $(\mathrm{n}=8)$. (b) Cell proliferation rates were determined using a WST assay. $X$ axis: days; $Y$ axis: OD value $(450 \mathrm{~nm})(\mathrm{n}=6)$. Cells in both regions were stimulated with PDGF $(25 \mu \mathrm{g} /$ $\mathrm{ml}$ ) for seven days and analysed by (c) Total collagen assay and (d) Sirius Red staining. Open circle, Vehicle $(\mathrm{n}=4)$; Closed circle, PDGF $(\mathrm{n}=4)$. Representative data are shown. (e) PDGF-stimulated cells at day 3 were analysed by qRT-PCR. Ligamentous-related genes: COL1, COL3, and TNC; Open circle, Vehicle $(\mathrm{n}=4)$; Closed circle, PDGF $(\mathrm{n}=4)$. (f) PDGF-stimulated cells at day 3 were analysed by immunofluorescence. Green, SMAalexa-488; Red, COL1-cy3; Blue, DAPI; Scale bar: $50 \mu \mathrm{m}$. Representative image data are shown $(\mathrm{n}=3)$. Error bars show standard error of the mean. ${ }^{*} p<0.05$.

differentiation with higher expression of $\mathrm{CD} 34^{+}$cells. On the other hand, the more proximal portion of the remnants had a stronger tendency for osteogenic and ligamentous differentiation. Characteristics of the ACL remnant tissue should be considered when performing remnant-preserving ACLR or harvesting ACL remnants for tissue engineering. 


\begin{abstract}
Materials and Methods
Patients. This study was carried out in accordance with institutional guidelines and approval from the Ethics Committee of Hanyang University Hospital, with written informed consent from all subjects (IRB-2018-05-001). Between March and June 2018, ruptured ACL remnants were extracted from 11 patients (8 males, 3 females) with a mean age of $23.8 \pm 4.69$ years (range, $17-31$ years), who had undergone primary ACL reconstruction within four weeks following injury. In case of a participant under age of 18 years, we had obtained parental consent. Patients $<40$ years of age who had a complete ACL rupture of femoral origin with at least $20 \mathrm{~mm}$ of intact ligament from the tibial insertion were included in the study. Remnants were extracted en-bloc, marked at the distal third (within $10 \mathrm{~mm}$ from the tibial insertion) and middle third (between 10-20 $\mathrm{mm}$ from the tibial insertion) and dissected using a number 11 blade. The most distal remnant at the tibial insertional area was preserved to prevent iatrogenic injury to the anterior root of the lateral meniscus. Supplementary table 1 shows the patient demographic data.
\end{abstract}

Isolation of human primary ACL remnant cells. The distal and middle thirds of the ACL remnant were each cut into 1 -cm or smaller segments with scissors. The segments were enzymatically digested with $1 \mathrm{mg} / \mathrm{ml}$ collagenase-type I (Gibco, 17100-017; Sigma, C0130) in serum-free DMEM containing penicillin-streptomycin antibiotics (Thermo Fisher, 15140122) and incubated at $37^{\circ} \mathrm{C}$ for $16-20$ hours. Cell suspensions were filtered through a nylon mesh, washed in serum-free DMEM several times, and seeded for culture.

Osteogenic, chondrogenic, and ligamentous differentiation. Osteogenic differentiation methods and activity assessments have been previously reported ${ }^{36}$. Briefly, cells from the distal and middle ACL regions were seeded in growth medium (DMEM with high glucose, Hyclone, SH30243.01) and then differentiated with osteogenic medium (ascorbic acid, dexamethasone, and $\beta$-glycerophosphate) into mature osteoblasts. Early stages of differentiation were assessed by ALP staining and activity. Late stages were assessed by quantification of alizarin red S (ARS) staining. For chondrogenic differentiation, cells were pelleted in DMEM/F-12 medium supplemented with infulin-transferrin-selenious acid (ITS) mixture, sodium pyruvate, ascorbate-2-phosphate, dexamethasone, and TGF $\beta 1$ in a $15-\mathrm{ml}$ conical tube ${ }^{37}$. Chondrogenic differentiation was assessed by toluidine blue and safranin O staining. For ligamentous differentiation, cells were stimulated with human PDGF-BB (PeproTech, 10014B) according to the indicated duration and dose $\mathrm{e}^{38,39}$. The ligamentous status of stimulated cells was assessed by Picro Sirius Red staining (Abcam, ab150681) and total collagen assay (BioVision, K218), according to the manufacturer's instructions. The capacity of ligamentous differentiation status was defined as collagen synthesis using Picro Sirus Red staining and total collagen assay and COL1, COL3, and TNC expression using qRT-PCR. Differentiation medium was changed every three days.

Cell proliferation assay. Cell proliferation was assessed using water-soluble tetrazolium salt (WST) (DoGen, EZ-1000), according to the manufacturer's instructions. Briefly, cells were seeded in 96-well plates at a density of $1 \mathrm{E} 10^{3}$ cells per well $(\mathrm{n}=3)$ with DMEM growth medium. WST solution was added directly to the cells as indicated, and the cells were then incubated for $1 \mathrm{~h}$ to allow the WST to metabolize to formazan. Absorbance was measured using a microplate plate reader at $450 \mathrm{~nm}$.

qRT-PCR and immunoblotting. RNA and protein extractions were performed as previously described ${ }^{40}$. RNA and proteins were extracted from stimulated cells with NucleoZOL and 1X RIPA buffer, respectively. Complementary DNA was generated from $1 \mu \mathrm{g}$ of total RNA with reverse transcriptase (Thermo Scientific, EP0442). The cells were lysed with 1X RIPA buffer containing phosphatase (Cell signaling, 5870 S) and protease (Calbiochem, 535140) inhibitors. Proteins were quantified with a Bradford assay. A total of 20-50 $\mu \mathrm{g}$ of protein was subjected to immunoblotting.

The qRT-PCR primers used are as follows: ALP forward, 5'-ACGAGCTGAACAGGAACAACGT-3'; ALP reverse, 5'-CACCAGCAAGAAGAAGCCTTTG-3'; RUNX2 forward, 5' -TGAGCTGAGAGGACATATGGCC-3'; RUNX2 reverse, $5^{\prime}$-TAGACACCAAACTCCACAGCCC-3' ${ }^{\prime}$ COL1 forward, $5^{\prime}$-AGTGGTTTGGATGGTGCCAA-3'; COL1 reverse, 5'-GCACCATCATTTCCACGAGC-3'; SOX9 forward, 5'-CTGAACGAGAGCGAGAAGCG-3'; SOX9 reverse, $5^{\prime}$-CCCGTTCTTCACCGACTTCC-3'; COL2 forward, 5'-CAACCAGGACCAAAGGGACA-3'; COL2 reverse, $5^{\prime}$-ACCTTTGTCACCACGATCCC-3'; COL3 forward, 5'- CTTCTCTCCAGCCGAGCTTC-3'; COL3 reverse, 5' - CCAGTGTGTTTCGTGCAACC-3'; TNC forward, 5'- GGTTGCTGGAGACTGTGGAA-3'; TNC reverse, $5^{\prime}$ - AGGTTTTCCAGAAGGGGCAG-3'; CD34 forward, 5'-CTCCAGCTGTGCGGAGTTTA-3'; CD34 reverse, 5'-TTGGCCAAGACCAGCAGTAG-3'; OCN forward, 5'-ATGAGAGCCCTCACACTCCT-3'; OCN reverse, $5^{\prime}$-CTTGGACACAAAGGCTGCAC-3'; ACAN forward, 5' -TGGGAACCAGCCTATACCCCAG-3'; ACAN reverse, $5^{\prime}$-CAGTTGCAGAAGGGCCTTCTGTAC- $3^{\prime}$

The antibodies for immunoblotting were as follows: SOX9 (Merck Millipore, AB5535), ALP (Santa, 365765), RUNX2 (Cell signaling, 12556), $\beta$-actin (Cell signaling, 3700), and GAPDH (Cell signaling, 2118).

Immunofluorescence (IF). Immunofluorescence was performed as previously described ${ }^{40}$. Briefly, cells were washed with 1X PBS (calcium and magnesium free) and fixed with $4 \%$ paraformaldehyde for $10 \mathrm{~min}$ at room temperature. Cells were then washed with 1X PBS three times. Cells were permeabilized in PBS containing $0.3 \%$ Triton X-100 and $10 \%$ BSA for $1 \mathrm{~h}$ and then washed three times with PBS. The cells were immunostained with primary antibodies diluted in PBS with $10 \%$ BSA at $4^{\circ} \mathrm{C}$ overnight. Next, cells were washed with $1 \mathrm{X}$ PBS for $10 \mathrm{~min}$ three times and incubated with CY3- or Alexa Fluor 488-labeled secondary antibodies for $1 \mathrm{~h}$. Nuclei were counterstained with 4, 6-diammidino-2-phenylindole (DAPI) (Vectashield, H1200). Immunofluorescence images were analysed by confocal microscopy (Leica Microsystems, Wetzlar, Germany). The antibodies for immunofluorescence are as follows: COL1A1 (Santa, 8784), SMA (Santa, 53142), SOX9 (Abcam, ab185966), and ALP (Santa, 365765). 
Fluorescence-activated cell sorting (FACS). Isolated cells were fixed with $2 \%$ formalin and stained with CD34-APC (Biolegend, 343607), CD90-PE (Biolegend, 328109), CD105-PE (Biolegend, 323205), CD146-APC (Biolegend, 361015), IgG2a-APC (Biolegend, 400221), or IgG1-PE (Biolegend, 400112) for $10 \mathrm{~min}$ at $4^{\circ} \mathrm{C}$. Following the staining, the cells were washed with $1 \mathrm{X}$ PBS containing $0.5 \%$ BSA and $0.1 \%$ sodium azide and analysed by flow cytometry (FACS Canto II, BD Biosciences).

Statistical analysis. Data were generated and analysed with a two-tailed paired t-test using GraphPad Prism 6 software. All data are expressed as mean \pm standard deviation from at least three independent experiments.

\section{Data availability}

The data that support the findings of this study are available from the corresponding author upon reasonable request.

Received: 24 September 2019; Accepted: 4 February 2020;

Published online: 20 February 2020

\section{References}

1. Duquin, T. R., Wind, W. M., Fineberg, M. S., Smolinski, R. J. \& Buyea, C. M. Current trends in anterior cruciate ligament reconstruction. J. Knee Surg. 22, 7-12 (2009).

2. Murray, M. M., Martin, S. D., Martin, T. L. \& Spector, M. Histological changes in the human anterior cruciate ligament after rupture. J. Bone Jt. Surg. Am. 82, 1387-1397, https://doi.org/10.2106/00004623-200010000-00004 (2000).

3. Agung, M. et al. Mobilization of bone marrow-derived mesenchymal stem cells into the injured tissues after intraarticular injection and their contribution to tissue regeneration. Knee Surg. Sports Traumatol. Arthrosc. 14, 1307-1314, https://doi.org/10.1007/s00167006-0124-8 (2006).

4. Holm, I., Oiestad, B. E., Risberg, M. A., Gunderson, R. \& Aune, A. K. No differences in prevalence of osteoarthritis or function after open versus endoscopic technique for anterior cruciate ligament reconstruction: 12-year follow-up report of a randomized controlled trial. Am. J. Sports Med. 40, 2492-2498, https://doi.org/10.1177/0363546512458766 (2012).

5. Lee, D. W., Shim, J. C., Yang, S. J., Cho, S. I. \& Kim, J. G. Functional Effects of Single Semitendinosus Tendon Harvesting in Anatomic Anterior Cruciate Ligament Reconstruction: Comparison of Single versus Dual Hamstring Harvesting. Clin. Orthop. Surg. 11, 60-72, https://doi.org/10.4055/cios.2019.11.1.60 (2019).

6. Kyung, H.-S. Graft considerations for successful anterior cruciate ligament reconstruction. Knee Surgery \& Related Research $\mathbf{3 1}$, https://doi.org/10.1186/s43019-019-0003-5 (2019).

7. Ballock, R. T., Woo, S. L., Lyon, R. M., Hollis, J. M. \& Akeson, W. H. Use of patellar tendon autograft for anterior cruciate ligament reconstruction in the rabbit: a long-term histologic and biomechanical study. J. Orthop. Res. 7, 474-485, https://doi.org/10.1002/ jor.1100070404 (1989).

8. Mehta, A. et al. Effects of Anteromedial Portal versus Transtibial ACL Tunnel Preparation on Contact Characteristics of the Graft and the Tibial Tunnel Aperture. Clin. Orthop. Surg. 11, 52-59, https://doi.org/10.4055/cios.2019.11.1.52 (2019).

9. Grana, W. A., Egle, D. M., Mahnken, R. \& Goodhart, C. W. An analysis of autograft fixation after anterior cruciate ligament reconstruction in a rabbit model. Am. J. Sports Med. 22, 344-351, https://doi.org/10.1177/036354659402200309 (1994).

10. Tohyama, H., Yoshikawa, T., Ju, Y. J. \& Yasuda, K. Revascularization in the tendon graft following anterior cruciate ligament reconstruction of the knee: its mechanisms and regulation. Chang. Gung Med. J. 32, 133-139 (2009).

11. Cobellis, G. et al. Beneficial effects of VEGF secreted from stromal cells in supporting endothelial cell functions: therapeutic implications for critical limb ischemia. Cell Transpl. 19, 1425-1437, https://doi.org/10.3727/096368910X509068 (2010).

12. Kalka, C. et al. Transplantation of ex vivo expanded endothelial progenitor cells for therapeutic neovascularization. Proc. Natl Acad. Sci. USA 97, 3422-3427, https://doi.org/10.1073/pnas.070046397 (2000).

13. Kawamoto, A. et al. Intramyocardial transplantation of autologous endothelial progenitor cells for therapeutic neovascularization of myocardial ischemia. Circulation 107, 461-468, https://doi.org/10.1161/01.cir.0000046450.89986.50 (2003).

14. Mifune, Y. et al. Tendon graft revitalization using adult anterior cruciate ligament (ACL)-derived CD34+ cell sheets for ACL reconstruction. Biomater. 34, 5476-5487, https://doi.org/10.1016/j.biomaterials.2013.04.013 (2013).

15. Mifune, Y. et al. Therapeutic potential of anterior cruciate ligament-derived stem cells for anterior cruciate ligament reconstruction. Cell Transpl. 21, 1651-1665, https://doi.org/10.3727/096368912X647234 (2012).

16. Matsumoto, T. et al. Reduction of tunnel enlargement with use of autologous ruptured tissue in anterior cruciate ligament reconstruction: a pilot clinical trial. Arthrosc. 30, 468-474, https://doi.org/10.1016/j.arthro.2013.12.014 (2014).

17. Matsumoto, T. et al. Acceleration of tendon-bone healing of anterior cruciate ligament graft using autologous ruptured tissue. Am. J. Sports Med. 40, 1296-1302, https://doi.org/10.1177/0363546512439026 (2012).

18. Ahn, G. Y. et al. Factors Affecting the Extent of Graft Tendon Synovialization after Double-Bundle Anterior Cruciate Ligament Reconstruction: Based on Second-Look Arthroscopic Findings. Clin. Orthop. Surg. 10, 413-419, https://doi.org/10.4055/ cios.2018.10.4.413(2018).

19. Petersen, W. \& Tillmann, B. Structure and vascularization of the cruciate ligaments of the human knee joint. Anat. Embryol. 200, 325-334, https://doi.org/10.1007/s004290050283 (1999).

20. Ahn, J. H. et al. Anterior cruciate ligament reconstruction using remnant preservation and a femoral tensioning technique: clinical and magnetic resonance imaging results. Arthrosc. 27, 1079-1089, https://doi.org/10.1016/j.arthro.2011.03.002 (2011).

21. Dhillon, M. S., Bali, K. \& Vasistha, R. K. Immunohistological evaluation of proprioceptive potential of the residual stump of injured anterior cruciate ligaments (ACL). Int. Orthop. 34, 737-741, https://doi.org/10.1007/s00264-009-0948-1 (2010).

22. Sun, L., Zhou, X., Wu, B. \& Tian, M. Inhibitory effect of synovial fluid on tendon-to-bone healing: an experimental study in rabbits. Arthrosc. 28, 1297-1305, https://doi.org/10.1016/j.arthro.2012.02.017 (2012).

23. Bray, R. C., Leonard, C. A. \& Salo, P. T. Vascular physiology and long-term healing of partial ligament tears. J. Orthop. Res. 20, 984-989, https://doi.org/10.1016/S0736-0266(02)00012-8 (2002)

24. Kodama, Y., Furumatsu, T., Hino, T., Kamatsuki, Y. \& Ozaki, T. Minimal Ablation of the Tibial Stump Using Bony Landmarks Improved Stability and Synovial Coverage Following Double-Bundle Anterior Cruciate Ligament Reconstruction. Knee Surg. Relat. Res. 30, 348-355, https://doi.org/10.5792/ksrr.18.024 (2018).

25. Zhang, S. et al. Anterior cruciate ligament remnant tissue harvested within 3-months after injury predicts higher healing potential. BMC Musculoskelet. Disord. 16, 390, https://doi.org/10.1186/s12891-015-0855-0 (2015).

26. Takazawa, Y. et al. ACL Reconstruction Preserving the ACL Remnant Achieves Good Clinical Outcomes and Can Reduce Subsequent Graft Rupture. Orthop. J. Sports Med. 1, 2325967113505076, https://doi.org/10.1177/2325967113505076 (2013).

27. Kim, M. K. et al. Comparison of second-look arthroscopic findings and clinical results according to the amount of preserved remnant in anterior cruciate ligament reconstruction. Knee 21, 774-778, https://doi.org/10.1016/j.knee.2014.02.011 (2014). 
28. Guo, L. et al. An Arthroscopic Second-Look Study on the Effect of Remnant Preservation on Synovialization of Bone-Patellar Tendon-Bone Allograft in Anterior Cruciate Ligament Reconstruction. Arthrosc. 32, 868-877, https://doi.org/10.1016/j. arthro.2015.11.017 (2016).

29. Noh, J. H., Kyung, H. S., Roh, Y. H. \& Kang, T. S. Remnant-preserving and re-tensioning technique to cover the graft in anterior cruciate ligament reconstruction. Knee Surg. Sports Traumatol. Arthrosc. 25, 1205-1210, https://doi.org/10.1007/s00167-015-38547 (2017).

30. Cole, M. A., Quan, T., Voorhees, J. J. \& Fisher, G. J. Extracellular matrix regulation of fibroblast function: redefining our perspective on skin aging. J. Cell Commun. Signal. 12, 35-43, https://doi.org/10.1007/s12079-018-0459-1 (2018).

31. Nakano, N. et al. Age-dependent healing potential of anterior cruciate ligament remnant-derived cells. Am. J. Sports Med. 43, 700-708, https://doi.org/10.1177/0363546514561436 (2015).

32. Naraoka, T. et al. Time-dependent gene expression and immunohistochemical analysis of the injured anterior cruciate ligament. Bone Jt. Res. 1, 238-244, https://doi.org/10.1302/2046-3758.110.2000118 (2012).

33. Kirizuki, S. et al. The Influence of Ruptured Scar Pattern on the Healing Potential of Anterior Cruciate Ligament Remnant Cells. Am. J. Sports Med. 46, 1382-1388, https://doi.org/10.1177/0363546518755753 (2018).

34. Prager, P. et al. Mesenchymal Stem Cells Isolated from the Anterior Cruciate Ligament: Characterization and Comparison of Cells from Young and Old Donors. Knee Surg. Relat. Res. 30, 193-205, https://doi.org/10.5792/ksrr.17.067 (2018).

35. Duthon, V. B. et al. Anatomy of the anterior cruciate ligament. Knee Surg. Sports Traumatol. Arthrosc. 14, 204-213, https://doi. org/10.1007/s00167-005-0679-9 (2006).

36. Jo, S. et al. Identification and characterization of human bone-derived cells. Biochem. Biophys. Res. Commun. 495, 1257-1263, https://doi.org/10.1016/j.bbrc.2017.11.155 (2018).

37. Herlofsen, S. R., Kuchler, A. M., Melvik, J. E. \& Brinchmann, J. E. Chondrogenic differentiation of human bone marrow-derived mesenchymal stem cells in self-gelling alginate discs reveals novel chondrogenic signature gene clusters. Tissue Eng. Part. A 17, 1003-1013, https://doi.org/10.1089/ten.TEA.2010.0499 (2011).

38. Hildebrand, K. A. et al. The effects of platelet-derived growth factor-BB on healing of the rabbit medial collateral ligament. An in vivo study. Am. J. Sports Med. 26, 549-554, https://doi.org/10.1177/03635465980260041401 (1998).

39. Batten, M. L., Hansen, J. C. \& Dahners, L. E. Influence of dosage and timing of application of platelet-derived growth factor on early healing of the rat medial collateral ligament. J. Orthop. Res. 14, 736-741, https://doi.org/10.1002/jor.1100140509 (1996).

40. Jo, S. et al. IL-17A induces osteoblast differentiation by activating JAK2/STAT3 in ankylosing spondylitis. Arthritis Res. Ther. 20, 115, https://doi.org/10.1186/s13075-018-1582-3 (2018).

\section{Acknowledgements}

This work was supported by the Basic Science Research Program through the National Research Foundation of Korea (NRF) funded by the Ministry of Science, ICT, \& Future (NRF-2016R1A2B4008606 and 2019R1A2C2004214 to T.K.) and the Ministry of Education (2017R1C1B5075203 to J.K.L. and 2017R1A6A3A11034394 to S.J.). The study was also supported by a Korea Health Technology R\&D grant through the Korea Health Industry Development Institute (KHIDI), which is funded by the Ministry of Health \& Welfare, Republic of Korea (HI17C0888 to T.K.).

\section{Author contributions}

Study conception and design: J.K.L., S.J. and T.K.; data collection: S.J., Y.L.L. and H.P.; data analysis and interpretation: J.K.L., S.J., J.S. and I.S.; drafting of the manuscript: J.K.L., S.J. and T.K.

\section{Competing interests}

The authors declare no competing interests.

\section{Additional information}

Supplementary information is available for this paper at https://doi.org/10.1038/s41598-020-60047-w.

Correspondence and requests for materials should be addressed to T.-H.K.

Reprints and permissions information is available at www.nature.com/reprints.

Publisher's note Springer Nature remains neutral with regard to jurisdictional claims in published maps and institutional affiliations.

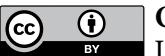

Open Access This article is licensed under a Creative Commons Attribution 4.0 International

License, which permits use, sharing, adaptation, distribution and reproduction in any medium or format, as long as you give appropriate credit to the original author(s) and the source, provide a link to the Creative Commons license, and indicate if changes were made. The images or other third party material in this article are included in the article's Creative Commons license, unless indicated otherwise in a credit line to the material. If material is not included in the article's Creative Commons license and your intended use is not permitted by statutory regulation or exceeds the permitted use, you will need to obtain permission directly from the copyright holder. To view a copy of this license, visit http://creativecommons.org/licenses/by/4.0/.

(C) The Author(s) 2020 